OS6 (1) - 2 (OS06W0033)

\title{
Measurement of the Fracture Toughness of Micrometer Scale Single Crystal Silicon by Tensile Test Method
}

\author{
Li Xueping, Dept. of Micro System Engineering, Nagoya Univ., Nagoya, Japan \\ Takashi Kasai, Dept. of Micro System Engineering, Nagoya Univ., Nagoya, Japan \\ Shigeki Nakao, Dept. of Micro System Engineering. Nagoya Univ., Nagoya, Japan \\ Hiroshi Tanaka, Dept. of Micro System Engineering, Nagoya Univ., Nagoya, Japan \\ Takeo Ando, Dept. of Micro System Engineering, Nagoya Univ., Nagoya, Japan \\ Mitsuhiro Shikida, Dept. of Micro System Engineering, Nagoya Univ., Nagoya, Japan \\ Kazuo Sato, Dept. of Micro System Engineering. Nagoya Univ., Nagoya, Japan
}

Mechanical properties of micrometer sized single crystal silicon material are one of the important factors for designing Micro-Electro-Mechanical Systems (MEMS) devices. Several types of testing system for measuring Young's modulus, fracture strength, and fracture toughness of MEMS materials have been proposed [1-3]. Ebrahimi et al proposed a fracture toughness measurement system, in which nano-indentation equipment was used for introducing micrometer-sized cracks on silicon planes. This method however is difficult to form the cracks with high-reproducibility. Fitzgerald et al used millimeter-sized specimen for measuring the fracture toughness [2].

We newly developed fracture toughness measurement system for micrometer-sized MEMS materials. We basically used "on-chip" device to measure the fracture toughness. The device has a function of specimen grip and a part of loading mechanism integrated with a film specimen. It means that the system does not need the manipulation of specimen. It is therefore suitable to measure the small and fragile notched specimen. We used Focused Ion Beam (FIB) process to obtain a micrometer-sized sharp notch with high reproducibility onto the specimen.

The width and thickness of the single-crystal-silicon specimen are 50 and $4.5 \mu \mathrm{m}$, respectively. There are two kinds of length of the specimen, 50 and $100 \mu \mathrm{m}$. The lengths of the notches are ranging from $0.1 \mu \mathrm{m}$ to several $\mu \mathrm{m}$. Considering the high aspect ratio of the width/thickness-size of the specimen, the thin film specimen is assumed in a uniform plane stress, where Griffith theory is applicable.

The stress intensity factor, $K$, is a function of the applied stress, crack length, and the correction factor as a function of the ratio of crack length to the width of the specimen. The fracture toughness, $K_{\mathrm{I}}$, is obtained by the stress intensity factor, $K$, when the specimen fractured. So we can calculate the $K_{\mathrm{IC}}$ from the measurement results.

The $K_{\mathrm{IC}}$ of silicon thin films were successfully calculated from the examination for the several chips. The obtained results are a little scattered ranging from 1.3 to $3.2 \mathrm{MPam}^{1 / 2}$. Comparing the $K_{\mathrm{IC}}$ of bulk silicon, 0.9 1.2 $\mathrm{MPam}^{1 / 2}$, the results show an apparent size effect, i.e., the values become larger for the smaller specimen.

References

1.F.Ebrahimi, L.Kalwani, Materials Science and Engineering A 268(1999)1 16-126

2.A.M.Fitzgerald, R.H.Dauskardt,T.W.Kenny, Sensors and Actuators 83(2000)194-199

3.K.Sato, T.Yoshioka, T.Ando, M.Shikida, T.Kawabata, Sensors And Actuators A,70(1998)148-152 